\title{
CurRiculum SEMANTIC RETRIEVAL SYSTEM BASED ON DISTANT SUPERVISION
}

\author{
Qingwen Tian, Shixing Zhou, Yu Cheng, Jianxia Chen, \\ Yi Gao and Shuijing Zhang
}

School of computer science, Hubei University of Technology, Hubei, China

\begin{abstract}
Knowledge Graph is a semantic network that reveals the relationship between entities, which construction is to describe various entities, concepts and their relationships in the real world. Since knowledge graph can effectively reveal the relationship between the different knowledge items, it has been widely utilized in the intelligent education. In particular, relation extraction is the critical part of knowledge graph and plays a very important role in the construction of knowledge graph. According to the different magnitude of data labeling, entity relationship extraction tasks of deep learning can be divided into two categories: supervised and distant supervised. Supervised learning approaches can extract effective entity relationships. However, these approaches rely on labeled data heavily resulting in the time-consuming and laborconsuming. The distant supervision approach is widely concerned by researchers because it can generate the entity relation extraction automatically. However, the development and application of the distant supervised approach has been seriously hindered due to the noises, lack of information and disequilibrium in the relation extraction tasks. Inspired by the above analysis, the paper proposes a novel curriculum points relationship extraction model based on the distant supervision. In particular, firstly the research of the distant supervised relationship extraction model based on the sentence bag attention mechanism to extract the relationship of curriculum points. Secondly, the research of knowledge graph construction based on the knowledge ontology. Thirdly, the development of curriculum semantic retrieval platform based on Web. Compared with the existing advanced models, the AUC of this system is increased by $14.2 \%$; At the same time, taking "big data processing" course in computer field as an example, the relationship extraction result with $F 1$ value of $88.1 \%$ is realized. The experimental results show that the proposed model provides an effective solution for the development and application of knowledge graph in the field of intelligent education.
\end{abstract}

\section{KEYWORDS}

Knowledge Graph, Curriculum Points, Distant Supervision, Relation Extraction, Sentence Bag Attention Mechanism, Ontology Construction.

\section{INTRODUCTION}

As the knowledge base of the Semantic Network, the knowledge graph (KG) is becoming one of the important education applications using artificial intelligence technology due to its significant capability[1][2][3][4][5]. In particular, relation extraction is an important part of information extraction technology. It refers to the modeling of text information to automatically extract the semantic relationship between entity pairs and extract effective semantic knowledge. It is a very critical part of the construction of knowledge graphs[6].

David C. Wyld et al. (Eds): SOFEA, CTCM, BIBC, SIPR, NCWC, CSEN, EDTECH - 2021

pp. 31-45, 2021. CS \& IT - CSCP 2021

DOI: $10.5121 /$ csit.2021.111603 
According to the difference of data set labeling magnitude, entity relationship extraction tasks of deep learning can be divided into two categories: supervised and distant supervised [7]. Supervised learning approaches can extract effective entity relationships. However, these approaches rely on labeled data heavily resulting in the time-consuming and labor-consuming. The distant supervision combines the advantages of semi-supervised learning and unsupervised learning and uses existing structured data to automatically annotate the data to generate training data [8]. In 2009, Mintz proposed the idea of distant supervision, that is, if two entities do not contain a certain relationship, all sentences involving these two entities represent the relationship, and the data for training the relationship extraction model can be automatically generated [9]. In 2016, Chris Quirk and Hoifung Poon proposed the first approach for applying distant supervision to cross sentence relation extraction, which can incorporate both standard dependencies and discourse relations, thus providing a unifying way to model relations within and across sentences [10]. However, the development and application of the distant supervised approach has been seriously hindered due to the noises, lack of information and disequilibrium in the relation extraction tasks.

Distant supervision methods based on deep learning are mainly improved network structures such as CNN, RNN and LSTM [11][12], such as the fusion method of PCNN and multi-example learning [13], and the fusion method of PCNN and attention mechanism [14]. In 2017, Ji et al. proposed a sentence-based attention mechanism and entity description, which reduced the noise problem and obtained semantic information in different sentences [15]. In 2018, Qu et al. proposed a relation extraction model of the word attention mechanism, which can improve the accuracy of relation extraction while reducing noise [16]. Fan et al. [17] solve the problems of sparseness and noise through matrix factorization and completion methods. In 2019, Craven et al.[18] proposed the idea of weakly supervised machine learning to extract the relationship between proteins and genes. However, for entity relationship extraction in a specific field, these research methods also need to solve the problem of domain knowledge base construction.

The KG is a series of different graphs that show the relationship between knowledge structure and the development process. It uses visualization technology to describe knowledge resources and carriers, excavates, analyses, and draws the connections between knowledge and them[19]. In general, $\mathrm{KG}$ is expressed by semantic standard language or ontology language. Ontology refers to a formal, clear and detailed description of a shared conceptual system[20]. The construction of the domain KG is usually based on the concept of domain knowledge ontology[21][22][23]. However, most of these systems only wants to achieve query expansion with the help of ontology, and does not combine semantic retrieval with $\mathrm{KG}$ to improve the quality and efficiency of retrieval.

Information retrieval refers to the process and technology of organizing information in a certain way and finding out relevant information according to the needs of information users [24]. The library information retrieval model proposed by $\mathrm{Bu}$ Yanyan creates ontology through standardization and planning, and processes user input requests through semantic logical reasoning, extraction, and query, and seeks ideal results from the knowledge base. It has a high recall rate and accuracy rate [25]. XiongWanying combined competitions and information literacy classes and proposed a curriculum content design based on information retrieval competitions to enrich the curriculum and enhance the teaching effect[26].Obviously, data retrieval and visual display are major trends in the development of information retrieval in the future.

Therefore, the paper proposes a novel Semantic Retrieval System model of "BigDataProcessing" course (BDP-SRS). In particular, the paper firstly proposes the system developed Distantly Supervised Relation Extraction Model based on Sentence Bags Attention (DSRE-SBA) to extract the relationship of curriculum points.Use statistics and deep learning methods to extract key 
words of course knowledge points, obtain knowledge point entities through domain entity optimization, and use remote supervision-based intra-pocket attention mechanism [27] to reduce the noise of the large amount of training data obtained, and then the denoised data is sent to Bi_LSTM (Bidirectional LSTM Networks with Entity-aware Attention using Latent Entity Typing) [28] for relation extraction. Secondly, the paper researches of knowledge graph construction based on the knowledge ontology. Thirdly, the paper developsa curriculum semantic retrieval platform based on Web. Compared with the existing models, the AUC of this system is increased by $14.2 \%$; At the same time, taking "big data processing" course in computer field as an example, the paper implements the relationship extraction result with F1 value of $88.1 \%$.

The main contents of the paper are as follows: Section 2 introduces the concepts of knowledge graph and semantic retrieval, as well as the method used in this paper; Section 3 mainly presents the overview of the system design, which mainly includes the design of two subsystems: relation extraction and knowledge graph semantic retrieval; Section 4 presents the experiment, comparative analysis of representative models and the implementation of the retrieval system; Section 5 is the conclusion.

\section{RELATED WORK}

\subsection{Knowledge Graph Construction}

KG is a knowledge base that represents entities (or concepts) and their relationships in the realworld in the form of graphs. There are two main ways to construct a KG: top-down approach and bottom-up approach. The top-down approach is to define the ontology and data schema for the KG and then add the entities to the knowledge base, which needs to utilize some existing structured knowledge base as its basic knowledge base. The bottom-up approach is to extract entities from some open linked data, select the entities with high confidence to join the knowledge base, and then construct the top-level ontology pattern. The flow chart of KG construction is shown in Figure 1.

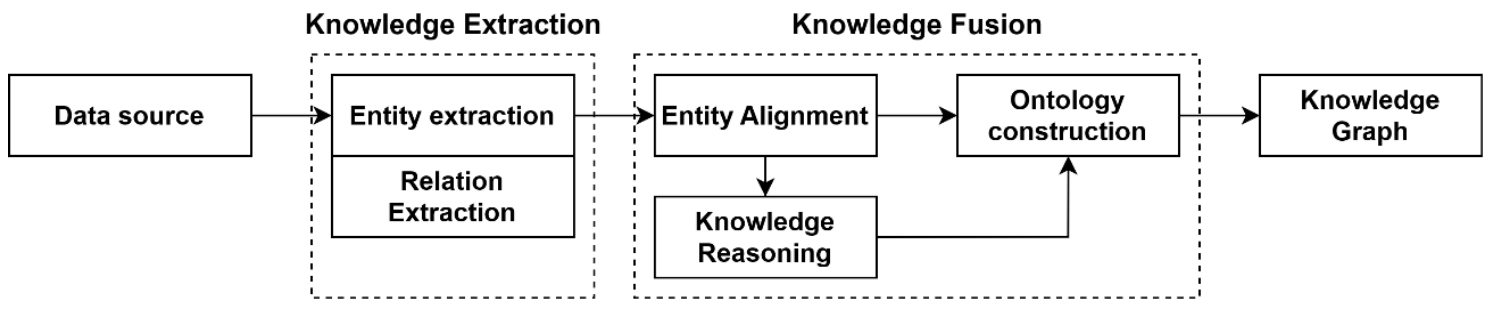

Figure 1. The flow chart of KG construction

As shown in Figure 1, there are three steps of KG construction including knowledge extraction, fusion and reasoning. Semi-structured and unstructured data have poor standardization, and it is difficult to obtain knowledge directly. Therefore, the entity extraction and relation extraction are needed to extract knowledge entities and relationships between entities, and then add them to the $\mathrm{KG}$. The construction process of KG is continuous and cyclic.

\subsection{Introduction to Semantic Retrieval}

Semantic retrieval enhances the conceptual understanding and analysis ability of the information retrieval system, understands and processes users' retrieval requests from the level of word meaning, and organizes information sources [29]. According to the retrieval effect and 
understanding degree, semantic retrieval can be divided into concept-based semantic retrieval and rule-based semantic retrieval [30].

- Concept-Based semantic retrieval. Through concept graph and attribute expansion based on the concept model in ontology, the search engine can accurately match the concept corresponding to the user's question, and then get the user's retrieval intention.

- Rule-Based semantic retrieval. The rule based on concept definition is utilized to judge whether or not the concept represented semantically is correct, and then form the inference function [31].

This paper adopts a concept-based semantic retrieval method. This is because its triples can represent the attributes and relationships of the concepts in the semantic layer.

\section{OVERVIEW OF SYSTEM DESIGN}

As shown in Figure 2, the semantic retrieval system model of "big data processing" course (BDPSRS) can be divided into the following two modules.

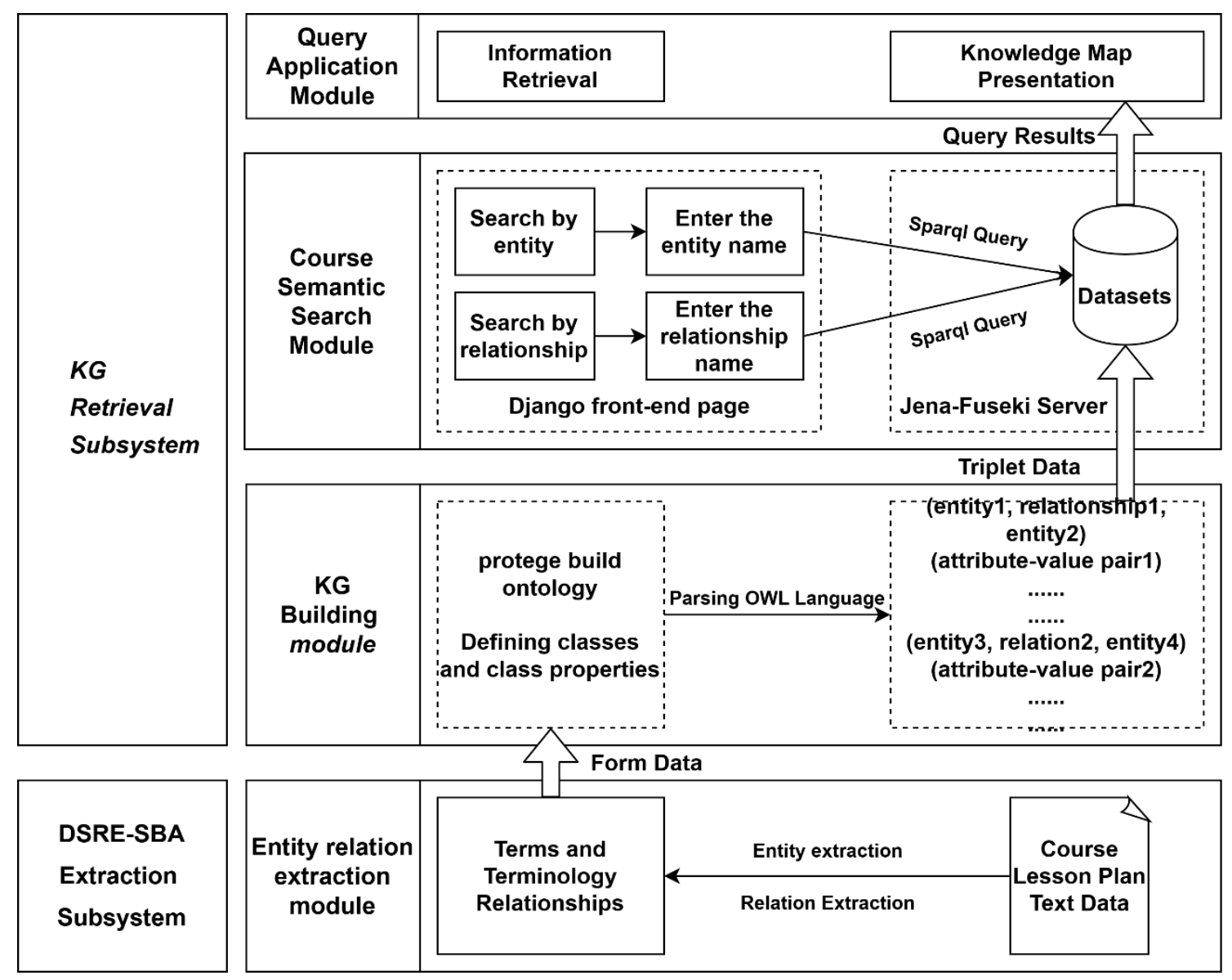

Figure 2. BDP-SRS Model Framework 


\subsection{Distantly Supervised Relation Extraction Model Based on Sentence Bag Attention}

In the relation extraction module, the system develops Distantly Supervised Relation Extraction Model based on Sentence Bags Attention (DSRE-SBA) to obtain data. As shown in Figure 3, DSRE-SBA model includes four parts: Extraction of curriculum knowledge entity; Build a large number of training data; Data noise reduction; Relation extraction.
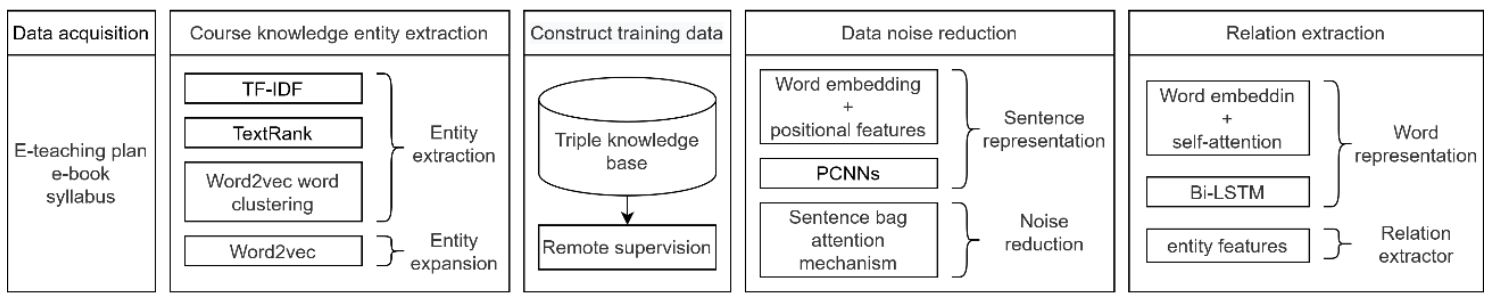

Figure 3. DSRE-SBA model frame diagram

\subsubsection{Course Knowledge Entity Extraction}

This model adopts Jieba, an open-source Chinese word segmentation tool, and constructs its word segmentation dictionary by using NLPIR Chinese stop word list and adding computer subject words. Then, the TF-IDF algorithm, TextRank algorithm, and Word2Vec word clustering are combined together to extract the curriculum knowledge entity keywords from the text that has been segmented.

\subsubsection{PCNNs-based Feature Extraction}

This model uses the neural network structure of PCNNs to automatically learn text features, instead of complex artificial features and feature processing procedures. Figure 4 is the model frame diagram of PCNN.

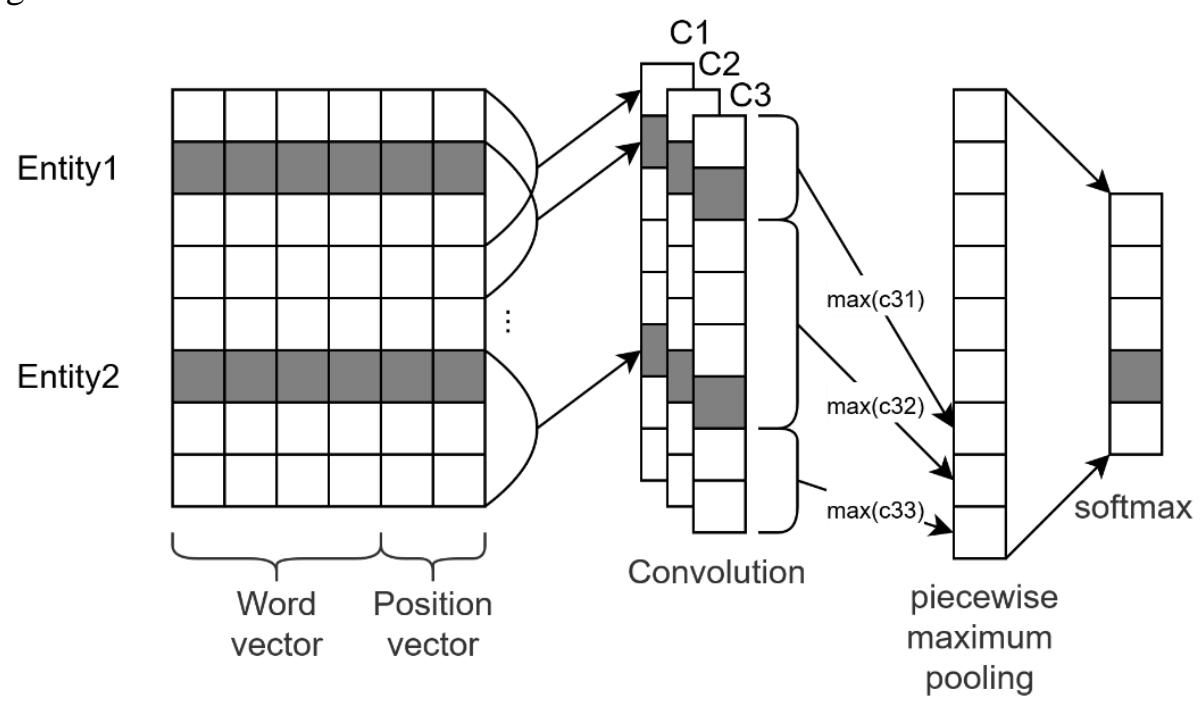

Figure 4. PCNN model frame diagram 


\subsubsection{Sentence Bag Attention Model}

The proposed sentence bag Attention model (SBA) utilized the similarity-based attention mechanism to express bag groups by weighting the bags., and took a relation-aware approach to calculate the weight of sentences in the each bag to represent a bag and set the noisy sentence to a smaller weight. The frame diagram of the SBA model is shown in Figure 5 the meaning of parameters is described as follows.

$g=\left\{b^{1}, b^{2}, \ldots, b^{n}\right\}$, represents a bag group, which contains multiple bags with the same relationship label marked by the distant supervisor, and $\mathrm{n}$ is the number of bags in a bag group.

$b^{i}=\left\{x_{1}^{i}, x_{2}^{i}, \ldots, x_{m_{i}}^{i}\right\}$ Means all sentences in a bag, and $m_{i}$ is the number of sentences in a bag bi.

$x_{j}^{i}=\left\{W_{j 1}^{i}, W_{j 2}^{i}, \ldots, W_{j l_{i j}}^{i}\right\}$ represents the $\mathrm{j}$ sentence in the $\mathrm{i}$ bag, and $l_{i j}$ is the length of the sentence.

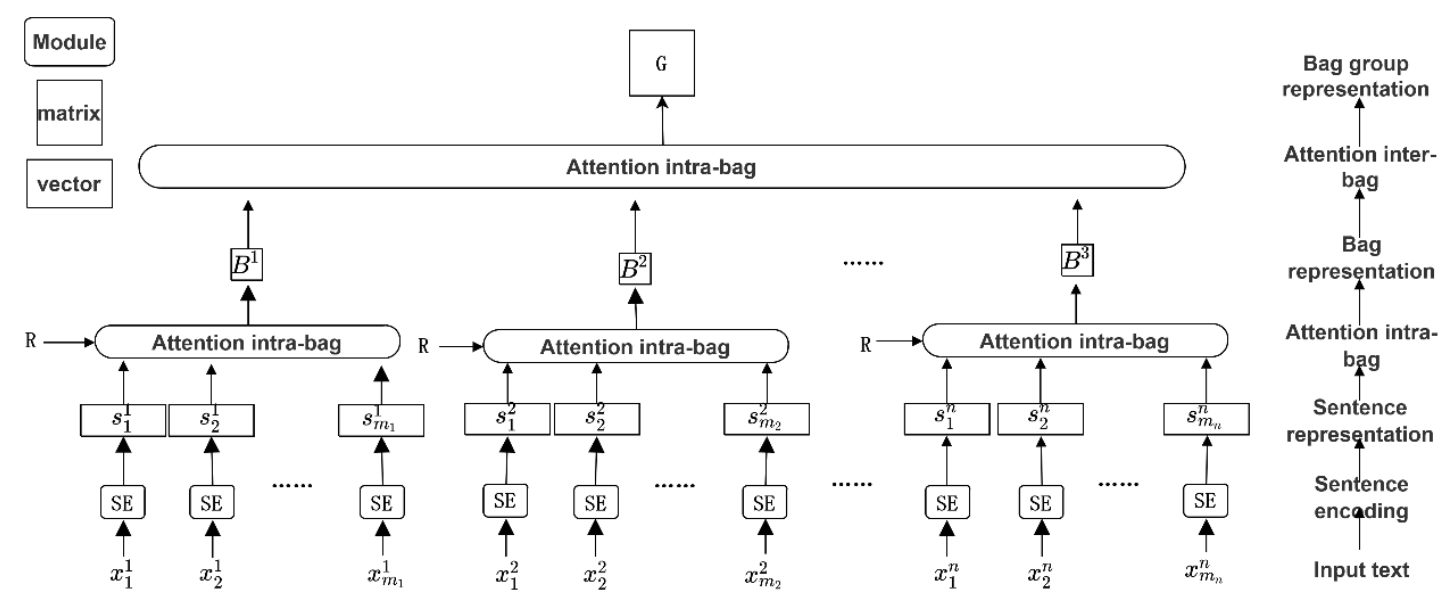

Figure 5. Sentence bag attention model frame diagram

\subsubsection{Bi_LSTM Based on Entity Perception Attention}

A Bidirectional LSTM Networks with Entity-Aware Attention (EA-BI_LSTM) model is proposed to classify the knowledge points of the course. This model is an end-to-end recursive neural model proposed by Joohong Lee et al. It combines an Entity perceptive attention mechanism with Latent Entity Typing[28]. The attention based on entity perception makes the model focus on the most important semantic information. The framework diagram of EABI_LSTM model is shown in Figure 6. 


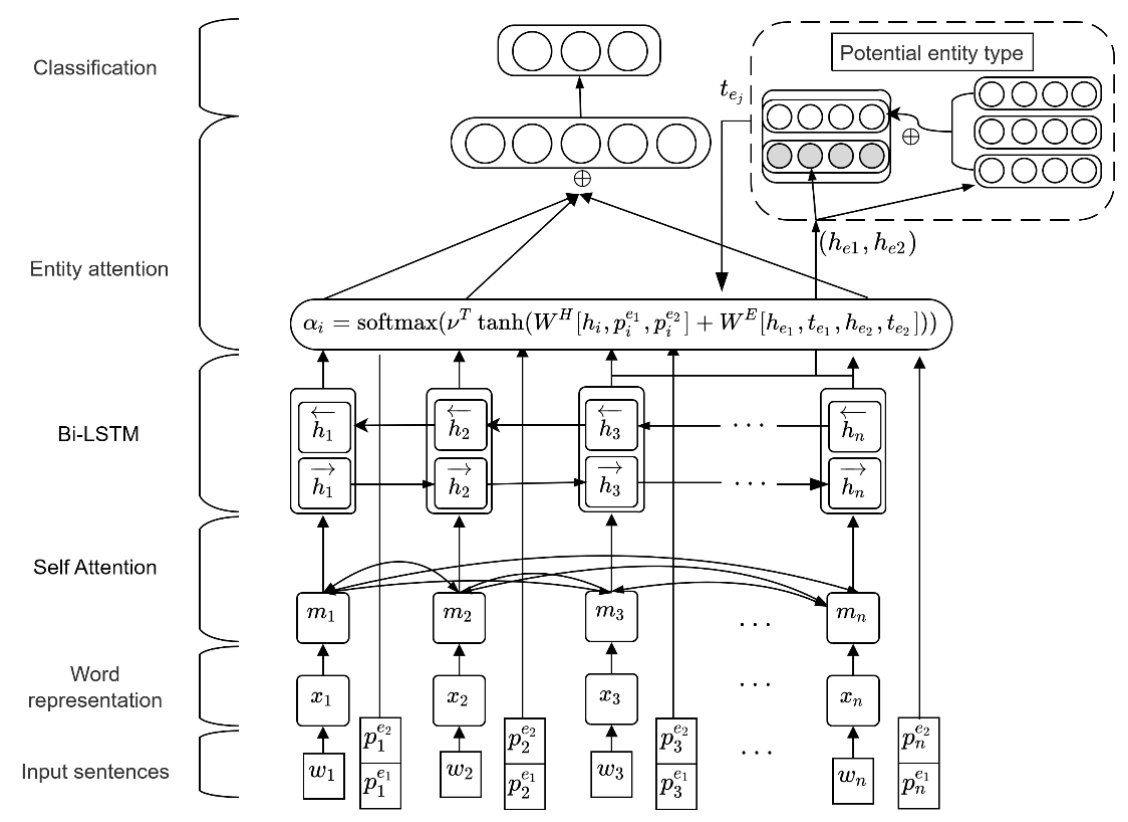

Figure 6. The framework diagram of EA-BI_LSTM model

The three characteristics of entity perception attention include:

- Hidden layer state of Bi_LSTMH $=\left\{\mathrm{h}_{1}, \mathrm{~h}_{2}, \ldots, \mathrm{h}_{\mathrm{n}}\right\}$.

- Relative location characteristics.

- Physical characteristics of LET. As shown in Formula (1)-(3).

$$
\begin{gathered}
u_{i}=\tanh \left(W^{H}\left[h_{i} ; p_{i}^{e_{1}} ; p_{i}^{e_{2}}\right]+W^{E}\left[h_{e_{1}} ; t_{1} ; h_{e_{2}} ; t_{2}\right]\right) \\
\alpha_{\mathrm{i}}=\frac{\exp \left(v^{T} u_{i}\right)}{\sum_{j-1}^{n} \exp \left(v^{T} u_{j}\right)} \\
z=\sum_{i=1}^{n} \alpha_{i} h_{i}
\end{gathered}
$$

\subsection{Knowledge Graph Retrieval Subsystem}

\subsubsection{Knowledge Graph Building Module}

The output data of DSRE-SBA subsystem is imported into Protege, which defines classes and class attributes in advance to construct the course knowledge ontology, and the course knowledge triad data containing entity relations and attributes are obtained. Finally, the query statement is deployed to the Django framework, and the KG is visualized by using the Echarts component. Figure 7 is the general framework of KG construction.

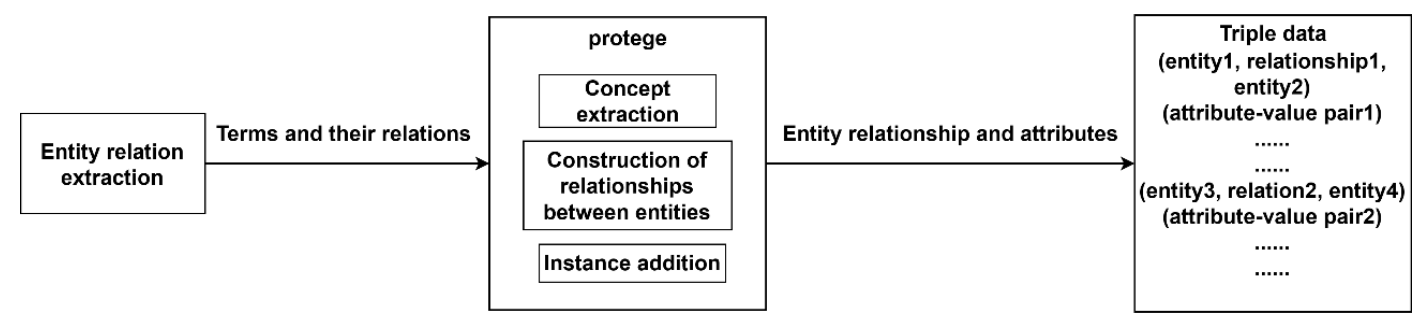

Figure 7. The general framework of KG construction. 


\subsubsection{Course Semantic Retrieval Module}

The semantic retrieval module adopts the front-end separation technology. The front-end simulated SPARQL request corresponding to the retrieval model and sent it to the back-end Apache Jena Fuseki server to query relevant data. After the data is processed, it is returned to the front-end, and the ontology is visualized by the Echarts component. Figure 8 is the schematic diagram of the semantic retrieval system.

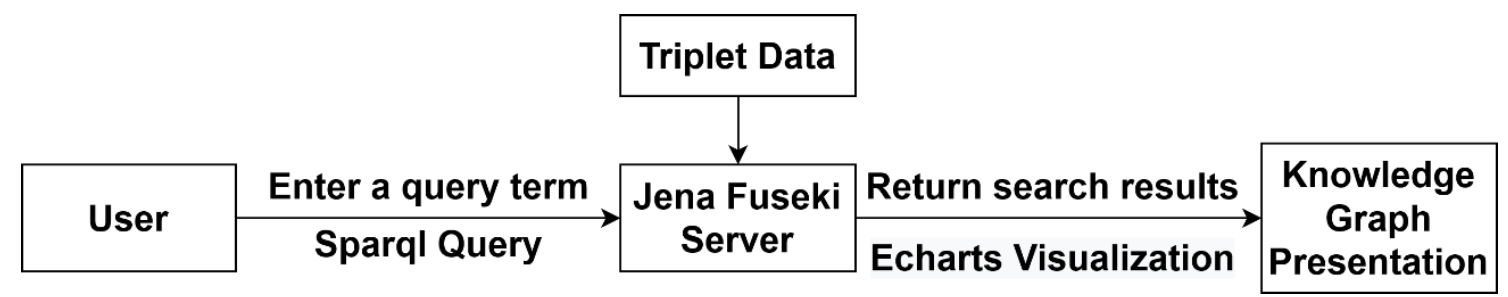

Figure 8 . The schematic diagram of semantic retrieval system

\subsubsection{Query Application Module}

Deploy the query statement on the Web framework Django, and use the Echarts component to visualize the query result. Figure 9 shows the query application page.

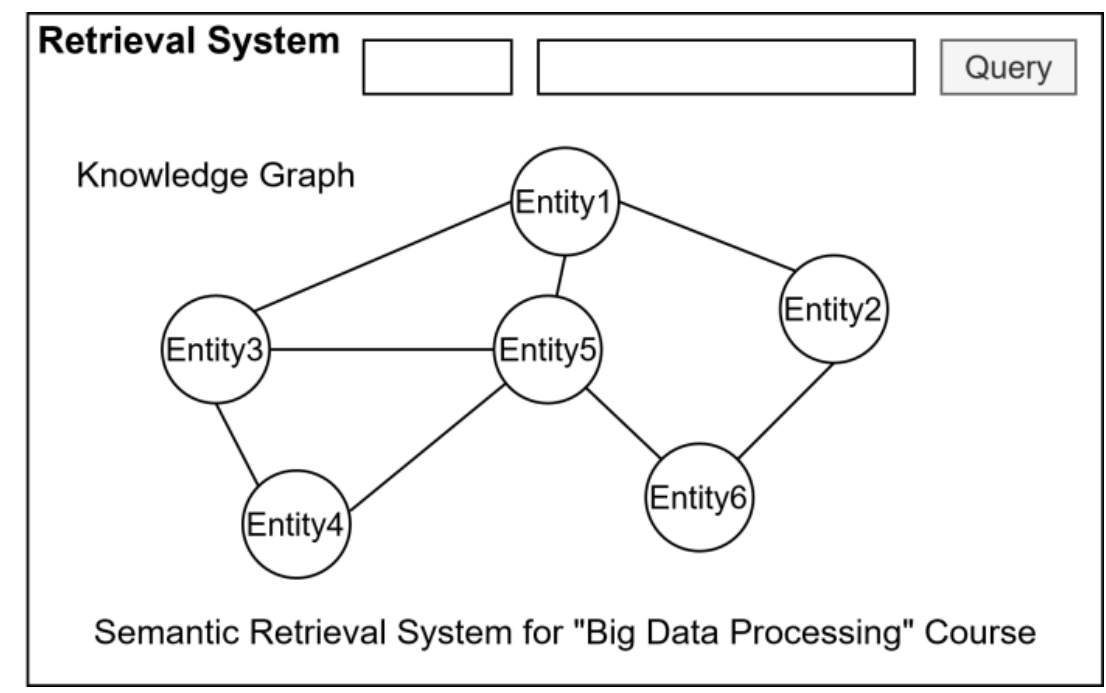

Figure 9. The query application page

\section{SYSTEM IMPLEMENTATION}

\subsection{Implementation of Entity Relation Extraction}

\subsubsection{Entity extraction}

Due to the limited data set acquired, the knowledge entities extracted are also limited, so the entity set needs to be expanded to ensure the integrity of the knowledge system. In this model, relevant teaching plans and books of "big data processing" courses are added into the wiki corpus to be trained as the training set of Word2 vec, and then a new round of manual optimization is carried out. English Entities Extension are shown in Table 1. 
Table 1. Example of an English entity extension result.

\begin{tabular}{|c|l|}
\hline Number & \multicolumn{1}{c|}{ English Entity } \\
\hline 1 & $\begin{array}{l}\text { map hadoop mapreduce job text class value new task tracker reducer key } \\
\text { mapper mapred public jobtracker context apache org string intwritableinput } \\
\text { records list tracker implements }\end{array}$ \\
\hline 2 & $\begin{array}{l}\text { output conf apilinux jar int throws task hdfs HDFS args void path import } \\
\text { max configuration streaming Streaming if xml static jvm line shuffle } \\
\text { extends http setmapperclass default uri missing }\end{array}$ \\
\hline
\end{tabular}

\subsubsection{Relation extraction}

According to the characteristics of knowledge, this model defines five kinds of relations between entities: description relation, leading and following relation, inclusion relation, parallel relation, and correlation relation. Then, a small knowledge base is built according to the five relationships, and the remote monitoring combined with the intra-bag attention mechanism is utilized for noise reduction. Finally, the relationship extractor is trained by Bi_LSTM. This knowledge base is automatically aligned with 10,728 unstructured texts, and 25,520 annotated data are obtained.

\subsubsection{Noise reduction experiment of sentence pouch attention mechanism}

In this paper, the sentence bag attention machine is used to reduce the noise of the data obtained by distant supervision. The experimental parameter settings are shown in Table 2 .

Table 2. Parameter setting of sentence bag model

\begin{tabular}{|c|c|c|}
\hline \multicolumn{2}{|c|}{ Parameter } & Value \\
\hline \multicolumn{2}{|c|}{ Word vector dimension } & 400 \\
\hline \multirow{2}{*}{$\begin{array}{c}\text { Location } \\
\text { feature }\end{array}$} & Maximum relative distance & 30 \\
\cline { 2 - 3 } CNN & Dimension & 5 \\
\cline { 2 - 3 } & Window size & 7 \\
\hline \multicolumn{2}{|c|}{ Feature maps } & 230 \\
\hline \multirow{2}{*}{ Batch size } & Dropout rate & 0.5 \\
\cline { 2 - 3 } & Learning rate & 0.1 \\
\hline \multicolumn{2}{|c|}{$N_{p}$} & 25 \\
\hline & Bag group size & 5 \\
\hline & Gradient clipping & 5.0 \\
\hline
\end{tabular}

This paper compares several examples of remote supervised noise reduction models. Table 3 shows the AUC values of different models. CNN and PCNN respectively represent the use of $\mathrm{CNN}$ or segmented CNN in the sentence encoder, ATT-BL represents the attention method proposed by (Lin and others, 2016)[32], ATT-RA represents the relational awareness in-bag attention method, while BAG-ATT represents the inter-bag attention method.

Table 3. AUC values of different models

\begin{tabular}{|l|l|}
\hline Model & AUC \\
\hline CNN+ATT-BL & 0.3478 \\
\hline CNN+ATT-BL+BAG-ATT & 0.3533 \\
\hline CNN+ATT-RA & 0.3773 \\
\hline CNN+ATT-RA+BAG-ATT & 0.3899 \\
\hline
\end{tabular}




\begin{tabular}{|l|l|}
\hline PCNN+ATT-BL & 0.3900 \\
\hline PCNN+ATT-BL+BAG-ATT & 0.3975 \\
\hline PCNN+ATT-RA & 0.4477 \\
\hline PCNN+ATT-RA+BAG-ATT & 0.4540 \\
\hline
\end{tabular}

For a more intuitive effect, Figure 10 compares the PR curve:

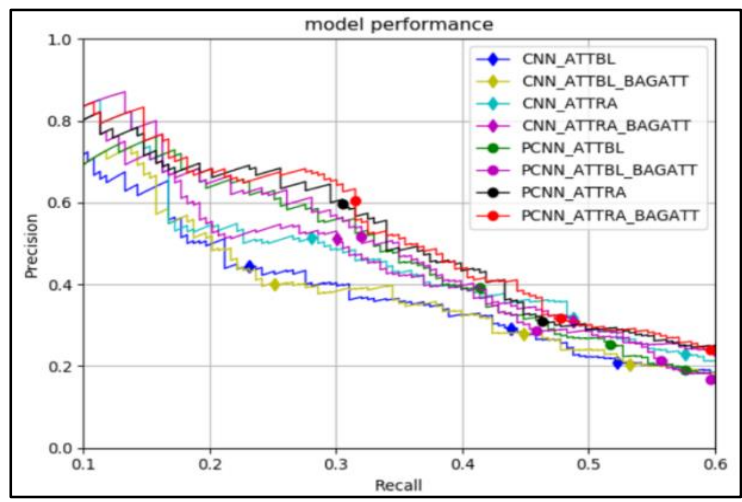

Figure 10. PR curves for different models

It can be seen from Figure 10 that the curves of PCNN_ATTRA and PCNN_ATTRA_BAGATT are basically above other curves, indicating that the in-bag and in-bag attention method is superior to the in-bag attention method proposed by Lin et al.

\subsubsection{Relation extraction experiment}

In this paper, the Bi-LSTM model based on entity perception concern is used for relation extraction, and the model method is tuned by $\mathrm{K}$-fold cross validation. The experimental parameters are shown in Table 4.

Table 4. Test parameter setting

\begin{tabular}{|c|c|c|}
\hline \multicolumn{2}{|l|}{ Parameter } & Value \\
\hline \multicolumn{2}{|c|}{ Word vector dimension } & 400 \\
\hline \multicolumn{2}{|c|}{ Position vector dimension } & 50 \\
\hline \multicolumn{2}{|c|}{ Number of potential entity types } & 3 \\
\hline \multicolumn{2}{|c|}{ Hidden layer size } & 400 \\
\hline \multicolumn{2}{|c|}{ Attention layer size } & 50 \\
\hline \multicolumn{2}{|l|}{ Batch size } & 20 \\
\hline \multicolumn{2}{|l|}{ Learning rate } & 1 \\
\hline \multirow{3}{*}{ Dropout Rate } & Word embedding layer & 0.3 \\
\hline & Bi_LSTM layer & 0.3 \\
\hline & Physical attention layer & 0.5 \\
\hline \multicolumn{2}{|c|}{ L2 regularization coefficient } & $10^{-5}$ \\
\hline
\end{tabular}


Table 5. F1_score for different models

\begin{tabular}{|l|l|l|}
\hline \multirow{2}{*}{ Model } & F1_score/(\%) \\
\cline { 2 - 3 } & $\begin{array}{l}\text { Undenoising } \\
\text { corpus }\end{array}$ & $\begin{array}{l}\text { Denoising } \\
\text { corpora }\end{array}$ \\
\hline CNN & 76.4 & 84.9 \\
\hline Attention-Bi_LSTM & 85.2 & 85.7 \\
\hline Attention-Bi_LSTM+LET & 86.4 & 88.1 \\
\hline
\end{tabular}

The experimental results of F1 of different models are shown in Table5. It can be seen that on the dataset of "big data processing" course, the sentence bag Attention mechanism still achieved certain effects, and the Attention-Bi_LSTM+LET model still performed the best.

\subsection{Construction and Implementation of KG}

Building KG based on domain knowledge ontology can be divided into three parts: concept extraction, Construction of relationships between entities and instance addition.

\subsubsection{Concept extraction}

The system customizes classes and attributes to build the curriculum ontology knowledge model. In the knowledge point of "big data processing" of this system, only one class MapReduce is designed.

\subsubsection{Construction of relationships between entities}

The relationship between entities in this system is divided into five types: Describe, Leading and succeeding, Contain, Parallel and Correlate.

\subsubsection{Instance addition}

In the process of constructing ontology, knowledge points need to be instantiated in the ontology knowledge model to realize relevant knowledge query.

\subsection{Implementation of Semantic Retrieval}

The system designs and implements the semantic retrieval system of the "big data processing" course, which is designed from two retrieval application modes of entity and relationship. This article uses Cellfie-ontology conversion domain specific language (MappingMaster DSL) to import excel table triple data to protégé. After completing the ontology construction, use the OntoGraf plug-in on protégé to display the overall ontology structure of the "Big Data Processing" course.

\subsubsection{Entity retrieval mode}

Take the entity "HDFS" as an example. Figure 11 shows the SPARQL query. 


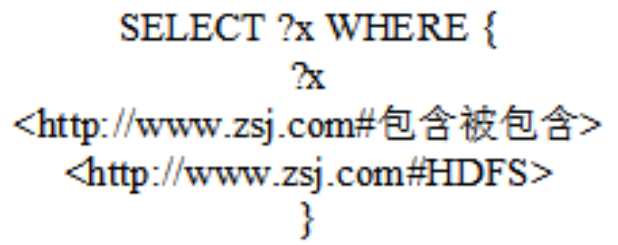

Figure 11. SPARQL query statement for "HDFS"

\subsubsection{Relational retrieval mode}

Take the relationship "Correlate" as an example. Figure 12 shows the SPARQL query statement.

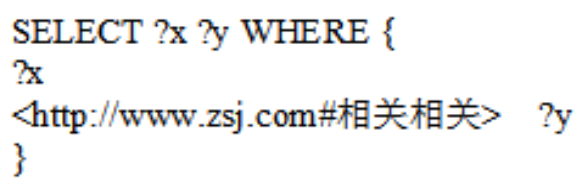

Figure 12. SPARQL query statement of "Relevant relevant"

\subsection{KG Display Implementation}

\subsubsection{Entity retrieval graph display}

Taking the entity "HDFS" as an example, Figure 13 shows the corresponding query result graph.

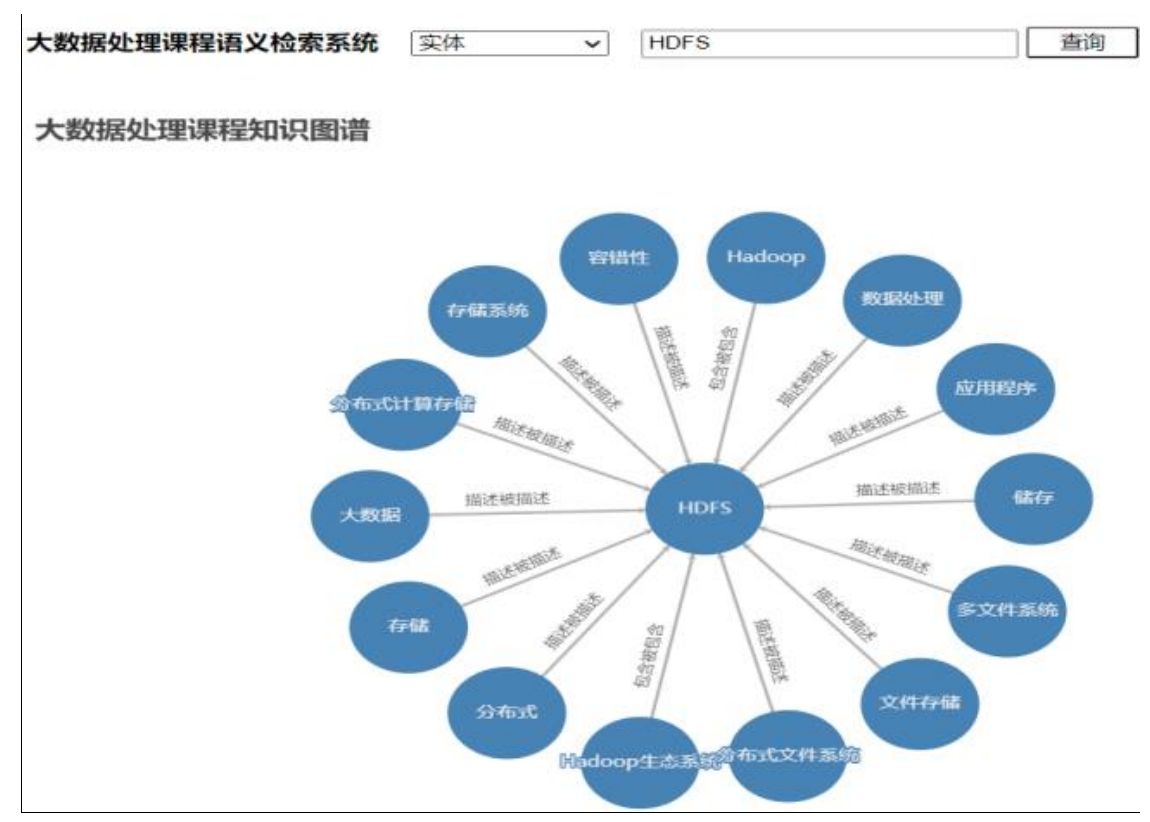

Figure 13. Relationship graph of "HDFS"

\subsubsection{Relational retrieval graph display}

Taking the relationship "Correlate" as an example, Figure 14 is the corresponding query result graph. 


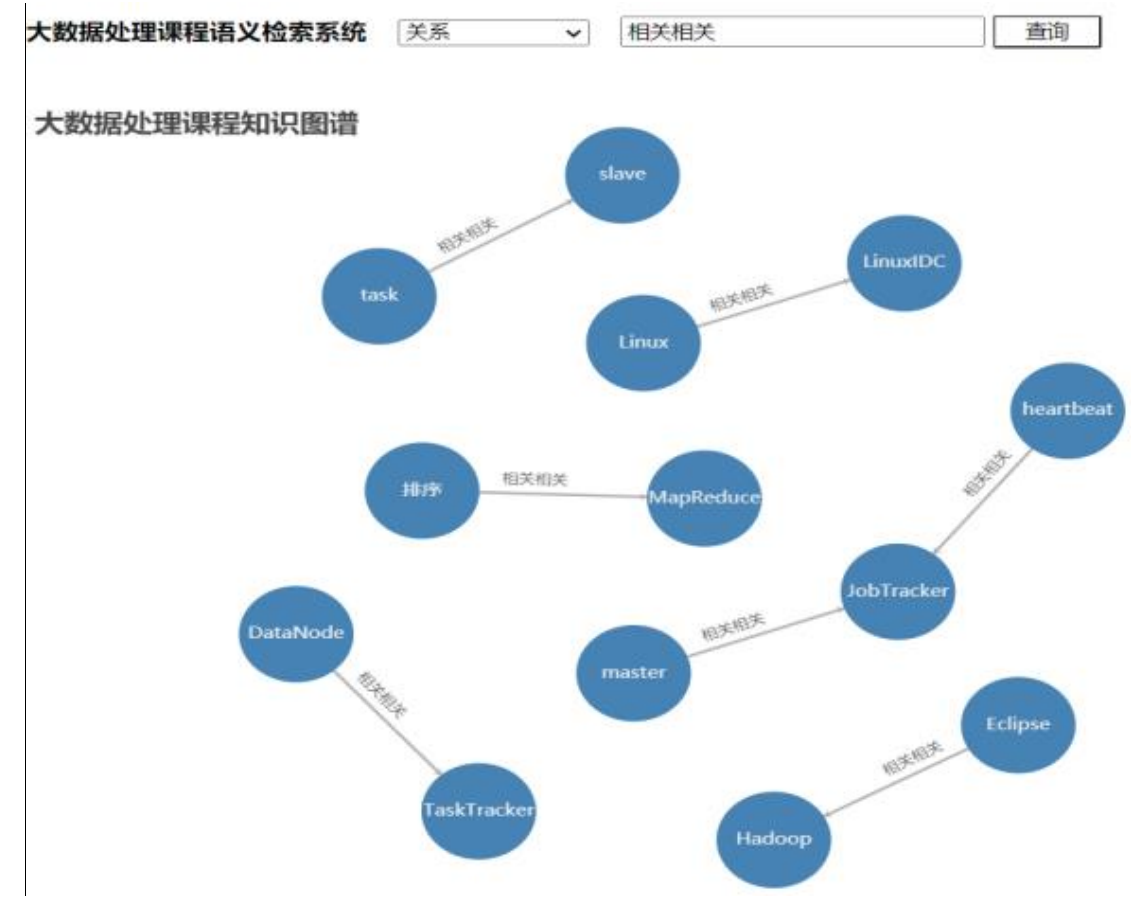

Figure 14. "Correlate" graph

\section{Conclusion}

This system extracts the knowledge points related to the "big data processing" course through the remote supervised relation extraction model DSRE-SBA based on sentence bag attention, constructs the course ontology knowledge model with the help of semantic ontology technology, extracts the terms and term relation data obtained through Cellfie import relation, and carries out semantic knowledge mining through SPARQL statement. Finally, the ontology knowledge model of "big data processing" course is designed and implemented by using two retrieval modes, entity, and relationship.

The limitation of this system lies in that only one class is created for the course knowledge points, and the non-hierarchical relation query is realized. Moreover, the limited course knowledge data leads to the incomplete ontology knowledge model of the "big data processing" course. On this basis, other course knowledge points will be added in the follow-up work to realize hierarchical relationship query, improve the course ontology, and establish a more comprehensive knowledge model of the course ontology.

\section{REFERENCES}

[1] Qi Guilin, Gao Huan \& Wu Tianxing. (2017). Research Progress of Knowledge Graph. Information Engineering (01).

[2] O'Donnell, Angela. (1996). Cognitive and Affective Outcomes of Learning from Knowledge Maps. Contemporary Educational Psychology - CONTEMP EDUC PSYCHOL. 21. 94-101. 10.1006/ceps.1996.0008.

[3] Bidarra, J. \&\& Dias, A. . (2004). Part iii-workshop on interactive visualisation and interaction technologiesecological strategies and knowledge mapping. Lecture Notes in Computer Science, 3036, 1025-1028.

[4] Suchanek, F. M. . (2007). YAGO : A core of semantic knowledge unifying WordNet and Wikipedia. Proceedings of the 16th International World Wide Web Conference, 2007.

[5] Lehmann J. DBpedia: A Nucleus for a Web of Open Data[C]// Semantic Web, International Semantic Web Conference, Asian Semantic Web Conference, Iswc + Aswc, Busan, Korea, November, (2007). 
[6] Chen JX, Yang F, Li C, et al. Research of Entity Relation Extraction Model Based on Dependency Parsing Neural Network[C]. the 15th International Conference on Natural Computation, Fuzzy Systems and Knowledge Discovery (ICNC-FSKD), 2019.

[7] E Haihong,ZhangWenjing,XiaoSiqi, et al.(2019) A review of deep learning entity relationship extraction research[J].Journal of Software,30(06):1793-1818.

[8] Yang Suizhu, Liu Yanxia, Zhang Kaiwen, Hong Yin, \& Huang Han. (2021).Overview of distant supervision relationship extraction. Chinese Journal of Computers, 44(8), 25.

[9] Mintz, M. , Bills, S. , Snow, R. L. , \&Jurafsky, D. S. . (2009). Distant supervision for relation extraction without labeled data. Tailieu Vn.

[10] Quirk, C. ,\& Poon, H. . (2016). Distant supervision for relation extraction beyond the sentence boundary.

[11] Jiao Licheng, Yang Shuyuan, Liu Fang, et al.(2016) Seventy years of neural networks: review and outlook[J].Chinese Journal of Computers,39(08):1697-1716.

[12] Zhou Feiyan, JinLinpeng, Dong Jun.(2017).Summary of Convolutional Neural Network Research[J].Chinese Journal of Computers,40(06):1229-1251.

[13] Zeng D, Liu K, Chen Y, Zhao J.(2015). Distant supervision for relation extraction via piecewise convolutional neural networks[C]. In: Proc. of the Conf. on Empirical Methods in Natural Language Processing, 1753-1762.

[14] Lin Y, Shen S, Liu Z, et al.(2016). Neural Relation Extraction with Selective Attention over Instances[C]// Proceedings of the 54th Annual Meeting of the Association for Computational Linguistics (Volume 1: Long Papers).

[15] Ji GL, Liu K, He SZ, et al.(2017). Distant supervision for relation extraction with sentence-level attention and entity descriptions[C]. In: Proc. of the AAAI, 3060-3066.

[16] Qu J, Ouyang D, Hua W, et al.(2018). Distant supervision for neural relation extraction integrated with word attention and property features[J]. Neural Networks, 100:59-69.

[17] Fan M, Zhao DL, Zhou Q, et al.(2014) Errata: Distant Supervision for Relation Extraction with Matrix Completion[J]. In arXiv, 1116094.

[18] Craven M ,Kumlien J (1999) Constructing biological knowledge bases by extracting information from text sources[C]// Proc Int Conf Intell Syst Mol Biol.

[19] Yao Ping, Li Kunwei, \& Zhang Yifan. (2020). Overview of Knowledge Graph Construction Technology. Information System Engineering.

[20] Gruber, T. R. . (1993). A translation approach to portable ontology specifications. Knowledge Acquisition, 5( 2), 199-220.

[21] OntoBroker, http://ontobroger.aifb.uni-karlsruhe.de [EB/OL], last accessed 2010/2/15.

[22] SKC, http://www.db.stanford.edu/ske [EB/OL], last accessed 2010/2/15.

[23] Song, J. , Zhang, W. , Xiao, W. , \& Tang, J. . (2005). Research on an ontology based information retrieval model. Journal of Nanjing University, 41(2).

[24] Wu Die. (2021). Research on visualization in the field of information retrieval in the past ten years [J]. Jiangsu Science and Technology Information, 21-23+29.

[25] Bu Yanyan. (2021). Library Information Retrieval Model Based on Artificial Intelligence Technology. Electronic Design Engineering.

[26] XiongWanying. (2021). Content design of information literacy course based on Information Retrieval Competition [J]. Encyclopedia Knowledge.

[27] Ye Z X , Ling Z H .(2019). Distant Supervision Relation Extraction with Intra-Bag and Inter-Bag Attentions[J].

[28] Joohong Lee, SangwooSeo, Yong Suk Choi.(2019). Semantic Relation Classification via Bidirectional LSTM Networks with Entity-Aware Attention Using Latent Entity Typing[J]. Symmetry,11(6).

[29] Cao Maocheng. Research on ontology-based semantic retrieval technology. (Doctoral dissertation, Shandong Institute of Light Industry).

[30] Tang Shouli. Research on Cloud Service Semantic Retrieval Model based on Ontology. (Beijing University of Aeronautics and Technology, Beijing)..

[31] Su Xiang. (2019). Research on the construction of "data structure" teaching resource platform based on knowledge graph. (Doctoral dissertation, Beijing Forestry University).

[32] Lin, Y., Shen, S. , Liu, Z. , Luan, H. , \& Sun, M. . (2016). Neural Relation Extraction with Selective Attention over Instances. Proceedings of the 54th Annual Meeting of the Association for Computational Linguistics (Volume 1: Long Papers). 


\section{AUTHORS}

Qingwen Tian An undergraduate student in the School of Computer Science, Hubei University of Technology.

Shixing Zhou An undergraduate student in the School of Computer Science, Hubei University of Technology.

Yu Cheng Associate professor of the School of Computer Science, Hubei University of Technology. Research direction: natural language processing and its application.

Jianxia Chen Associate professor and master tutor of the School of Computer Science, Hubei University of Technology. Research direction: cloud computing and big data, knowledge graph and natural language processing, intelligent planning research.

Yi Gao An undergraduate student in the School of Computer Science, Hubei University of Technology.

Shuijing Zhang Graduate student of Computer Science and Technology, Xidian University.
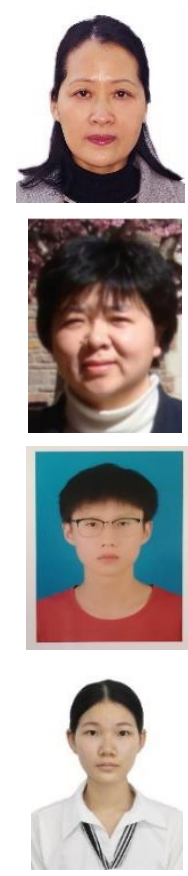

(C) 2021 By AIRCC Publishing Corporation. This article is published under the Creative Commons Attribution (CC BY) license. 